\title{
Linking nutrient loading, local abiotic variables, richness and biomasses of macrophytes, and associated invertebrate species in the north- eastern Baltic Sea
}

\author{
Jonne Kotta ${ }^{\bowtie}$ and Tiia Möller \\ Estonian Marine Institute, University of Tartu, Mäealuse 14, 12618 Tallinn, Estonia \\ $\bowtie$ Corresponding author, jonne@sea.ee
}

Received 5 May 2014, revised 5 July 2014, accepted 29 July 2014

\begin{abstract}
We studied relationships between nutrient loading, local abiotic variables, richness and biomasses of macrophytes, and associated invertebrate species in the north-eastern Baltic Sea. The study showed that nutrient load strongly correlated to the richness and biomass of macroalgal and invertebrate species and functions and often interacted with local abiotic variables such as salinity and depth. Generally elevated nutrient loads increased the species richness of macrophytes and benthic invertebrates and the biomass of annual macroalgae and of the majority of invertebrate feeding groups, but reduced the biomass of perennial macroalgae. The study also showed that the effect of nutrient loading was scale-specific, i.e. different responses to nutrients were observed at water-body and gulf scales. The biomass of the majority of species and functions was a function of the gulf-scale nutrient loading whereas the variability of annual algae and chironomidae reflected changes in the water-body level nutrient loading.
\end{abstract}

Key words: eutrophication, macrophytes, benthic invertebrates, abiotic environment, Baltic Sea.

\section{INTRODUCTION}

In order to establish standards for ecological conditions in groundwater, streams, lakes, and coastal areas most European countries have to implement the EU Water Framework Directive (WFD) by 2015. The aims of the WFD are to protect, enhance, and restore all water bodies and to obtain good water quality (European Union, 2000). For implementing the WFD it is necessary to establish efficient methods to link the ecological status with catchment loading.

Nutrient loading is considered to be the main process that causes changes in the structure and functioning of many coastal sea ecosystems (Elmgren, 1989; Duarte, 1995). Similarly, an elevated nutrient loading is a key risk for the Baltic Sea ecosystem shown by significant shifts in community structure associated to a 
dramatic increase in nutrient concentrations in recent decades (Kotta et al., 2000; Elmgren, 2001; Rönnberg, 2001; Bonsdorff et al., 2002; Grall and Chauvaud, 2002; Kotta and Witman, 2009).

Phytobenthos is regarded as a good early-warning indicator of the environmental status because they are stationary and visible by the naked eye. It is known that increasing availability of inorganic nutrients stimulates the abundance of ephemeral macroalgae and causes the dominance shift from perennial to annual macroalgae in shallow coastal waters (Sand-Jensen and Borum, 1991; Duarte, 1995; Valiela et al., 1997). It is therefore expected that ephemeral species are especially good indicators of water quality. However, other environmental variables such as substrate type and salinity may modulate the relationships between nutrient loading and ephemeral macroalgae (Eriksson and Bergström, 2005; Kotta et al., 2009).

Benthic invertebrate communities represent an intermediate trophic level and nutrient additions affect them in many ways. Increasing nutrient loads enhance the production of benthic and/or pelagic microalgae (Granéli and Sundbäck 1985; Howarth, 1988) and, hence, increase the amount of available food for benthic grazers, suspension feeders, and deposit feeders and ultimately for carnivores. As a consequence, abundance and growth responses of invertebrates are observed at moderate levels of nutrients (Posey et al., 1999; Kotta and Ólafsson, 2003; Lauringson and Kotta, 2006; Lauringson et al., 2007). Further addition of nutrients leads to hypoxia, appearance of ammonia and hydrogen sulphide, and consequently to the disappearance of benthic invertebrates (Gray et al., 2002; Kotta et al., 2007).

To date, we still lack scientific evidence for the nutrient-macrophyte-invertebrate relationship in order to adequately assess the water quality in the Baltic Sea region. Such a relationship can be found, however, when the correlation structure between environmental variables and biotic patterns is analysed in the multivariate space and non-linearities are taken into account. Multivariate analysis enables us to distinguish separate and interactive effects of nutrient loading and to estimate the relative contribution of nutrient loading to the overall variability of macrophyte communities. Besides, novel machine learning techniques such as Boosted Regression Trees (BRT) modelling also enables fitting complex nonlinear relationships. Avoiding overfitting the data, BRT provide very robust estimates. What is most important in the ecological perspective it automatically handles interaction effects between predictors. Consequently, such analysis can inform us about the relative contribution of different environmental variables to the variability of biotic patterns as well as to compute the functional form relationship between the environment (e.g. nutrient load) and biota, independent of other environmental variables. Due to its strong predictive performance, BRT is increasingly used in ecology (Elith et al., 2008).

We analysed links between nutrient loading and phytobenthic community structure and sought whether and how local abiotic variables modulated the nutrient-phytobenthos relationship in the north-eastern Baltic Sea. Our specific 
aim was to evaluate whether the biomasses of ephemeral macroalgae, deposit feeders, suspension feeders, herbivores, and carnivores were stimulated by the elevated load of nutrients in the north-eastern Baltic Sea. We also evaluated if the biomass of perennial or annual macroalgae could be a promising tool for the assessment of water quality.

\section{MATERIAL AND METHODS}

\section{Monitoring areas}

Sampling was performed on three transects in a frame of the Estonian National Marine Monitoring Programme in 1995-2005 (Fig. 1). The main characteristics of the transects are presented in Table 1.

The Eru transect is located at the southern shore of the Gulf of Finland and belongs to Lahemaa National Park. Salinity varies there from 5.1 to 5.6 PSU, which is typical of the central Gulf of Finland. Boulders, gravel, and sand prevail in the substrate. Although the area is relatively sheltered, strong currents and storms can occasionally displace large amounts of sediments and therefore change the structure of the bottom substrate. The local nutrient discharges and freshwater inflow are very low in the area.

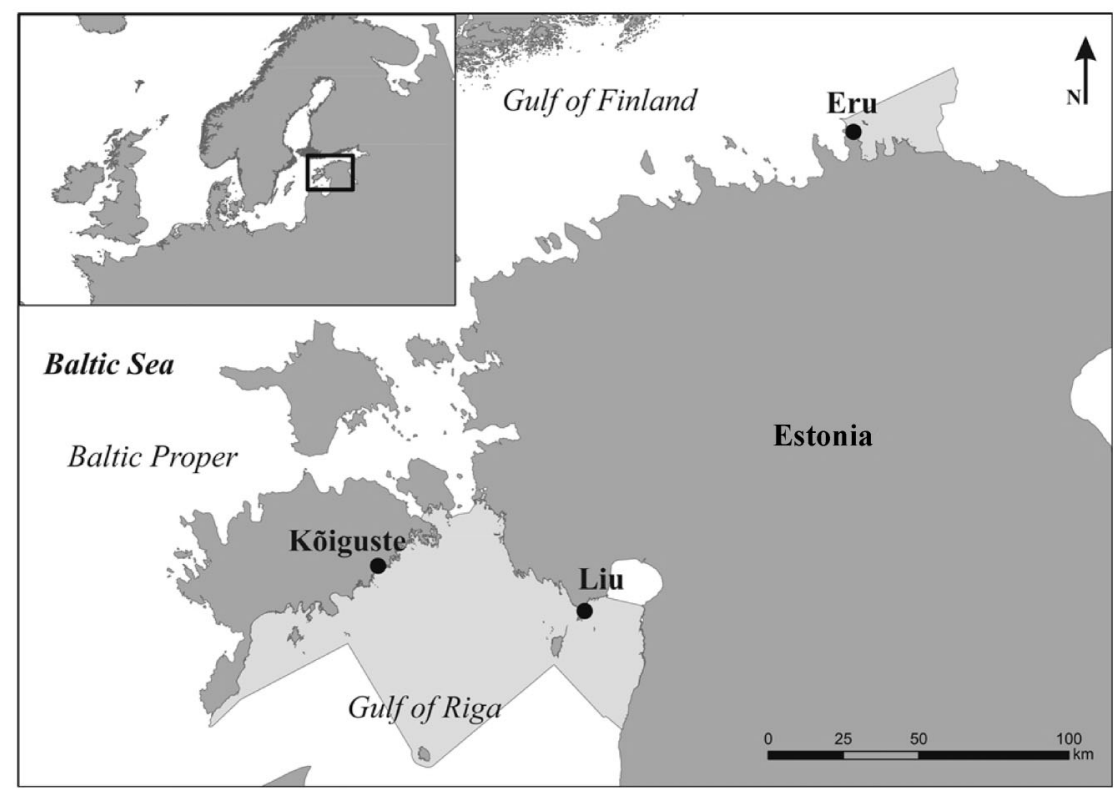

Fig. 1. Location of the studied areas in the Baltic Sea. Transects are indicated by filled circles and the studied water bodies by light grey. 


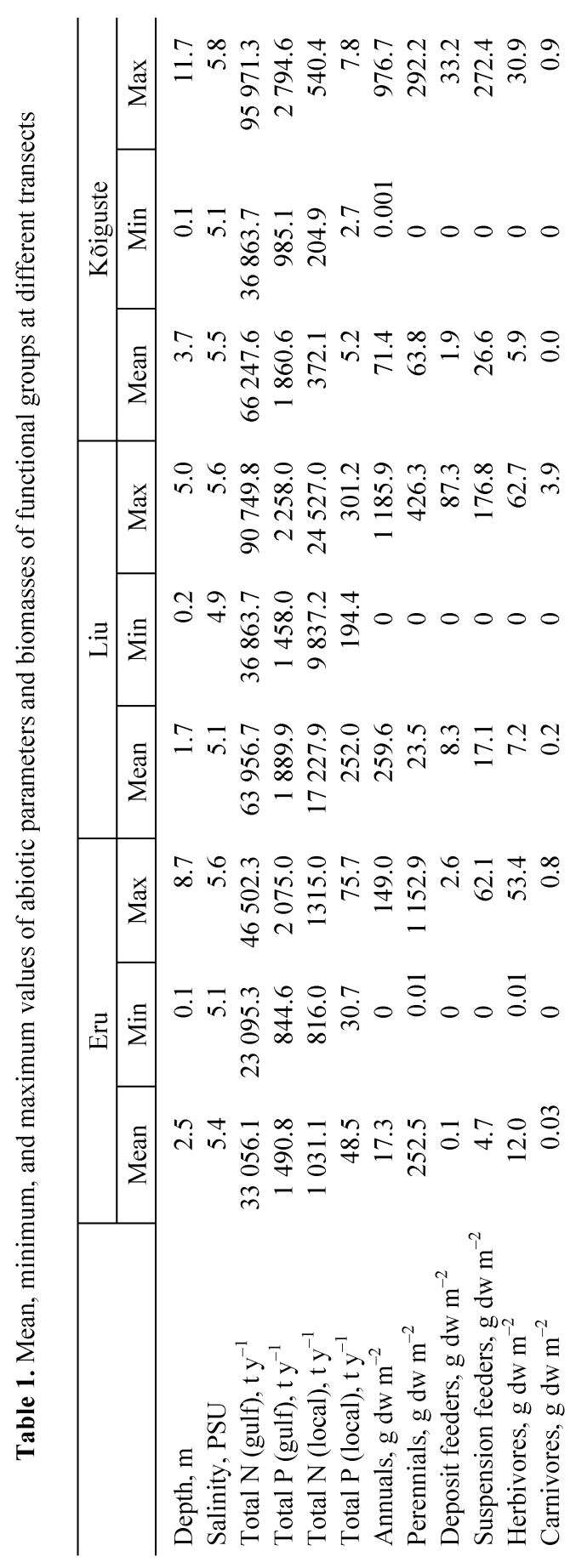


The Liu transect is situated at the outermost but sheltered part of Pärnu Bay, north-eastern Gulf of Riga. The bay is severely eutrophicated due to the loading from the Pärnu River (Kotta et al., 2008). Salinity varies between 4.9 and 5.6 PSU. The bottom consists mainly of sand and gravel mixed with clay. Occasionally some boulders are found.

The Kõiguste transect is located at the moderately exposed part of the northern Gulf of Riga. The area is influenced by a diffused nutrient load from the offshore regions of the Gulf of Riga. Salinity varies between 5.1 and 5.8 PSU. The bottom consists of boulders and gravel and is covered by fine sediment in the deeper areas.

\section{Data collection}

The phytobenthos monitoring methods follow the guidelines provided by HELCOM (2009). Sampling was carried out annually in the late summers of 1995-2005. Samples were collected from different transects as follows: in the Kõiguste transect during 1995-2005, at Liu in 1995 and 1999-2005, and at Eru in 1997-2005. During sampling the depth was recorded (a proxy of light condition) and sediment characteristics were described. Samples were taken with a metal frame $(20 \mathrm{~cm} \times 20 \mathrm{~cm})$ in three random replicates from depths $0.1,0.3,0.5$, and $1 \mathrm{~m}$ and deeper down as triplicates from depth intervals $1-2,2-4,4-6,6-8$, and 8-10 m.

In the laboratory algae and invertebrates were sorted to the species level and dry weight (per $1 \mathrm{~m}^{2}$ ) was found after drying the material at $60^{\circ} \mathrm{C}$ for $2-3$ weeks. Invertebrates were grouped according to their feeding type on the basis of literature and field observations as follows: herbivores, deposit feeders, suspension feeders, and carnivores (Bonsdorff and Pearson, 1999). Then the values were averaged over replicates for each transect and depth strata and were used for further statistical analyses.

Average salinity values were obtained from the results of hydrodynamical model calculations from the summers of 1995-2005. The calculations were based on the COHERENS model, which is a primitive equation ocean circulation model. It was formulated with spherical coordinates on a $1^{\prime} \times 1^{\prime}$ minute horizontal grid and 30 vertical sigma layers. The model was forced with hourly meteorological fields of $2 \mathrm{~m}$ air temperature, wind speed, wind stress vector, cloud cover, and relative humidity. The meteorological fields were obtained from an operational atmospheric model. The model was validated against water level, temperature, salinity, and water velocity measurements from the study area (the database of the Estonian Marine Institute) (Bendtsen et al., 2009). The data on the annual point source and riverine loads of total nitrogen and total phosphorus to the study area at local (i.e. water-body) level and gulf (i.e. regional loads) scales in 1995-2005 were obtained from the Estonian Ministry of Environment. 


\section{Data analysis}

Multivariate data analyses on the abiotic environment and biotic patterns were performed by the statistical program PRIMER version 6.1.5 (Clarke and Gorley, 2006). Invertebrate biomass data were not transformed. Similarities between each pair of samples were calculated using a zero-adjusted Bray-Curtis coefficient. The coefficient is known to outperform most other similarity measures and enables samples containing no organisms at all to be included (Clarke et al., 2006). The statistical differences in macrophyte and associated invertebrate assemblages between transects were obtained by the ANOSIM permutation test (Clarke and Green, 1988; Clarke, 1993). The contribution of each species to dissimilarities was investigated using the similarities percentages procedure (SIMPER) (Clarke, 1993).

In addition, the contribution of different environmental variables to the biomass of key macrophyte and invertebrate species as well as the aggregated community variables such as richness, total biomass, biomass of specific functional group was explored using the Boosted Regression Trees (BRT) technique. Prior to the analyses we checked if the studied environmental variables were highly intercorrelated. In general the results suggested that multicollinearity was never a severe issue in our data sets. BRT models are capable of handling different types of predictor variables and their predictive performance is superior to most traditional modelling methods. BRT models iteratively develop a large ensemble of small regression trees constructed from random subsets of the data. Each successive tree predicts the residuals from the previous tree to gradually boost the predictive performance of the overall model. In the BRT models all studied environmental variables were regressed to predict the functional form relationship between environmental variables and species/function biomasses. In fitting a BRT the learning rate and the tree complexity must be specified. The learning rate determines the contribution of each successive tree to the final model, as it proceeds through the iterations. The tree complexity fixes whether only main effects (tree complexity $=1$ ) or interactions are also included (tree complexity $>1$ ). Ultimately, the learning rate and tree complexity combined determine the total number of trees in the final model. For each species, multiple models were run varying both the model learning rate (between 0.1 and 0.001 ) and the number of trees (between 1000 and 10000 ). Then the optimum model was selected based on model performance. Typically, optimal learning rates, number of trees, and interaction depth were $0.01,2000$, and 5 , respectively. A random $20 \%$ of the data was assigned for testing model accuracy. The BRT modelling was done in the statistical software R using the gbm package (R Core Team, 2013).

\section{RESULTS}

Altogether 40 taxa of macrophytes and 47 taxa of benthic invertebrates were identified. Species richness was the highest in Kõiguste Bay; 35 plant and 44 
invertebrate taxa were recorded in the area over the period of ten years. Altogether 33 plant and 38 invertebrate taxa were recorded at Liu and 22 and 33 taxa at Eru, respectively (Table 2). The ephemeral algae Ceramium tenuicorne, Cladophora glomerata, and Pilayella littoralis dominated in the study area. Besides, the perennial species Furcellaria lumbricalis and Fucus vesiculosus and the higher plant Myriophyllum spicatum were widespread. These six species also contributed most to the dissimilarities in macroalgal communities among transects (ANOSIM $r=0.399, p<0.001$; SIMPER). The most common invertebrate taxa were Cerastoderma glaucum, Chironomidae, Gammarus spp., Idotea spp., and Theodoxus fluviatilis. However, species related to the differences among transects were primarily Mytilus trossulus, T. fluviatilis, and Dreissena polymorpha (ANOSIM $r=0.401, \mathrm{p}>0.001$; SIMPER).

The BRT modelling described a significant proportion of variability in the richness and biomass of benthic macrophyte and invertebrate species and

Table 2. Percent occurrence (\%) and average biomass (B) \pm SD of macroalgal and invertebrate species in the studied transects. Functional groups are denoted as follows: A - annual, $\mathrm{P}$ - perennial, $\mathrm{S}$ - suspension feeder, $\mathrm{C}$ - carnivore, $\mathrm{H}$ - herbivore, $\mathrm{D}$ - deposit feeder

\begin{tabular}{|c|c|c|c|c|c|c|c|}
\hline \multirow[t]{2}{*}{ Taxon } & \multirow[t]{2}{*}{ Function } & \multicolumn{2}{|r|}{ Eru } & \multicolumn{2}{|r|}{ Liu } & \multicolumn{2}{|r|}{ Kõiguste } \\
\hline & & $\%$ & $\mathrm{~B}$ & $\%$ & $\mathrm{~B}$ & $\%$ & $\mathrm{~B}$ \\
\hline $\begin{array}{l}\text { Aglaothamnion roseum } \\
\text { (Roth) Maggs \& } \\
\text { L'Hardy-Halos }\end{array}$ & A & & & & & 4 & $<0.01$ \\
\hline $\begin{array}{l}\text { Ceramium tenuicorne } \\
\text { (Kützing) Waern }\end{array}$ & A & 80 & $2.20 \pm 4.63$ & 68 & $0.39 \pm 0.74$ & 84 & $2.07 \pm 5.49$ \\
\hline $\begin{array}{l}\text { Ceramium virgatum } \\
\text { Roth }\end{array}$ & $\mathrm{P}$ & 5 & $0.01 \pm 0.09$ & 9 & $0.04 \pm 0.16$ & 7 & $<0.01$ \\
\hline $\begin{array}{l}\text { Chara aspera } \\
\text { Willdenow }\end{array}$ & A & & & 18 & $1.36 \pm 5.84$ & 17 & $0.67 \pm 2.16$ \\
\hline Chara baltica Bruzelius & A & & & 3 & $0.01 \pm 0.08$ & 1 & $0.01 \pm 0.09$ \\
\hline $\begin{array}{l}\text { Chara canescens } \\
\text { Desvaux \& Loiseleur }\end{array}$ & A & 2 & $<0.01$ & 12 & $0.09 \pm 0.40$ & 8 & $0.18 \pm 0.89$ \\
\hline $\begin{array}{l}\text { Chara connivens } \\
\text { Salzmann ex } \\
\text { A. Braun }\end{array}$ & A & & & 9 & $0.06 \pm 0.29$ & 1 & $0.05 \pm 0.43$ \\
\hline $\begin{array}{l}\text { Chara tomentosa } \\
\text { Linnaeus }\end{array}$ & A & & & 3 & $<0.01$ & & \\
\hline Chara Linnaeus & A & & & 6 & $0.03 \pm 0.14$ & 3 & $0.12 \pm 1.05$ \\
\hline $\begin{array}{l}\text { Chorda filum } \\
\text { (Linnaeus) } \\
\text { Stackhouse }\end{array}$ & A & 2 & $<0.01$ & & & 11 & $0.55 \pm 3.61$ \\
\hline $\begin{array}{l}\text { Chroodactylon ornatum } \\
\text { (C. Agardh) Basson }\end{array}$ & A & & & & & 3 & $0.01 \pm 0.04$ \\
\hline $\begin{array}{l}\text { Cladophora glomerata } \\
\text { (Linnaeus) Kützing }\end{array}$ & A & 57 & $8.46 \pm 16.92$ & 68 & $1.70 \pm 4.47$ & 66 & $7.04 \pm 20.15$ \\
\hline
\end{tabular}


Table 2. Continued

\begin{tabular}{|c|c|c|c|c|c|c|c|}
\hline \multirow[t]{2}{*}{ Taxon } & \multirow[t]{2}{*}{ Function } & \multicolumn{2}{|r|}{ Eru } & \multicolumn{2}{|r|}{ Liu } & \multicolumn{2}{|r|}{ Kõiguste } \\
\hline & & $\%$ & $\mathrm{~B}$ & $\%$ & $\mathrm{~B}$ & $\%$ & $\mathrm{~B}$ \\
\hline $\begin{array}{l}\text { Cladophora rupestris } \\
\text { (Linnaeus) Kützing }\end{array}$ & $\mathrm{P}$ & 27 & $0.61 \pm 3.60$ & 15 & $0.40 \pm 2.26$ & 12 & $0.26 \pm 1.95$ \\
\hline $\begin{array}{l}\text { Coccotylus truncatus } \\
\text { (Pallas) M. J. Wynne } \\
\text { \& J. N. Heine }\end{array}$ & $\mathrm{P}$ & 2 & $<0.01$ & 9 & $<0.01$ & 57 & $0.19 \pm 0.34$ \\
\hline $\begin{array}{l}\text { Dictyosiphon } \\
\text { foeniculaceus } \\
\text { (Hudson) Greville }\end{array}$ & A & 27 & $1.45 \pm 4.37$ & 6 & $<0.01$ & 34 & $4.18 \pm 28.64$ \\
\hline $\begin{array}{l}\text { Elachista fucicola } \\
\text { (Velley) Areschoug }\end{array}$ & A & 25 & $1.07 \pm 4.33$ & & & 7 & $<0.01$ \\
\hline $\begin{array}{l}\text { Fucus vesiculosus } \\
\text { Linnaeus }\end{array}$ & $\mathrm{P}$ & 70 & $237.00 \pm 326.01$ & 15 & $4.33 \pm 20.48$ & 36 & $21.40 \pm 51.03$ \\
\hline $\begin{array}{l}\text { Furcellaria lumbricalis } \\
\text { (Hudson) } \\
\text { J. V. Lamouroux }\end{array}$ & $\mathrm{P}$ & 27 & $3.41 \pm 9.73$ & 36 & $0.85 \pm 3.25$ & 92 & $23.55 \pm 39.85$ \\
\hline $\begin{array}{l}\text { Myriophyllum spicatum } \\
\text { Linnaeus }\end{array}$ & A & 2 & $<0.01$ & 77 & $29.92 \pm 59.39$ & 21 & $0.05 \pm 0.27$ \\
\hline $\begin{array}{l}\text { Percursaria percursa } \\
\text { (C. Agardh) } \\
\text { Rosenvinge }\end{array}$ & $\mathrm{P}$ & & & 3 & $<0.01$ & & \\
\hline $\begin{array}{l}\text { Pilayella littoralis } \\
\quad \text { (Linnaeus) Kjellman }\end{array}$ & A & 7 & $0.32 \pm 177$ & 6 & $0.03 \pm 0.12$ & 9 & $0.23 \pm 0.90$ \\
\hline $\begin{array}{l}\text { P. littoralis/Ectocarpus } \\
\text { siliculosus (Dillwyn) } \\
\text { Lyngbye }\end{array}$ & A & 59 & $3.37 \pm 10.38$ & 33 & $18.77 \pm 63.89$ & 87 & $45.92 \pm 117.68$ \\
\hline $\begin{array}{l}\text { Polysiphonia fibrillosa } \\
\text { (Dillwyn) Sprengel }\end{array}$ & A & 30 & $0.19 \pm 0.59$ & 18 & $0.12 \pm 0.39$ & 13 & $0.03 \pm 0.23$ \\
\hline $\begin{array}{l}\text { Polysiphonia fucoides } \\
\text { (Hudson) Greville }\end{array}$ & $\mathrm{P}$ & 30 & $1.74 \pm 5.65$ & 76 & $2.12 \pm 3.57$ & 70 & $3.46 \pm 6.08$ \\
\hline $\begin{array}{l}\text { Potamogeton } \\
\quad \text { perfoliatus Linnaeus }\end{array}$ & A & & & 47 & $1.40 \pm 3.27$ & 4 & $0.03 \pm 0.16$ \\
\hline $\begin{array}{l}\text { Ranunculus peltatus } \\
\text { Schrank subsp. } \\
\text { baudotii (Godr.) } \\
\text { Meikle ex } \\
\text { C. D. K. Cook }\end{array}$ & A & & & 3 & $<0.01$ & & \\
\hline $\begin{array}{l}\text { Rhizoclonium riparium } \\
\text { (Roth) Kützing ex } \\
\text { Harvey }\end{array}$ & A & & & & & 5 & $0.02 \pm 0.17$ \\
\hline $\begin{array}{l}\text { Rhodomela } \\
\quad \text { confervoides } \\
\text { (Hudson) P. C. Silva }\end{array}$ & $\mathrm{P}$ & 16 & $0.26 \pm 0.91$ & 3 & $<0.01$ & 63 & $1.54 \pm 3.14$ \\
\hline $\begin{array}{l}\text { Ruppia maritima } \\
\text { Linnaeus }\end{array}$ & A & & & 24 & $12.99 \pm 46.99$ & 25 & $3.96 \pm 14.00$ \\
\hline $\begin{array}{l}\text { Ruppia cirrhosa } \\
\text { (Petagna) Grande }\end{array}$ & A & & & 3 & $<0.01$ & & \\
\hline $\begin{array}{l}\text { Schoenoplectus } \\
\quad \text { tabernaemontanii } \\
\text { (C. C. Gmel.) Palla }\end{array}$ & A & & & & & 1 & $0.05 \pm 0.43$ \\
\hline
\end{tabular}


Relationship between nutrient loading and benthos

Table 2. Continued

\begin{tabular}{|c|c|c|c|c|c|c|c|}
\hline \multirow[t]{2}{*}{ Taxon } & \multirow[t]{2}{*}{ Function } & \multicolumn{2}{|r|}{ Eru } & \multicolumn{2}{|r|}{ Liu } & \multicolumn{2}{|r|}{ Kõiguste } \\
\hline & & $\%$ & $\mathrm{~B}$ & $\%$ & $\mathrm{~B}$ & $\%$ & $\mathrm{~B}$ \\
\hline $\begin{array}{l}\text { Sphacelaria arctica } \\
\text { Harvey }\end{array}$ & $\mathrm{P}$ & 36 & $9.48 \pm 55.42$ & 50 & $0.32 \pm 1.32$ & 83 & $13.36 \pm 17.65$ \\
\hline $\begin{array}{l}\text { Stictyosiphon tortilis } \\
\text { (Ruprecht) Reinke }\end{array}$ & A & 2 & $0.03 \pm 0.18$ & 3 & $<0.01$ & 47 & $2.37 \pm 5.45$ \\
\hline $\begin{array}{l}\text { Stuckenia pectinata (L.) } \\
\text { Böerner }\end{array}$ & A & & & 59 & $15.92 \pm 32.39$ & 16 & $0.31 \pm 1.42$ \\
\hline $\begin{array}{l}\text { Zannichellia palustris } \\
\text { Linnaeus }\end{array}$ & $\mathrm{A}$ & 2 & $0.01 \pm 0.07$ & 42 & $1.37 \pm 3.61$ & 37 & $2.93 \pm 11.57$ \\
\hline $\begin{array}{l}\text { Zostera marina } \\
\text { Linnaeus }\end{array}$ & $\mathrm{P}$ & 2 & $<0.01$ & 15 & $<0.01$ & 8 & $0.01 \pm 0.07$ \\
\hline $\begin{array}{l}\text { Tolypella nidifica } \\
\text { (O. F. Müller) } \\
\text { Leonhardi }\end{array}$ & A & & & 12 & $0.07 \pm 0.34$ & 28 & $0.27 \pm 0.91$ \\
\hline $\begin{array}{l}\text { Ulothrix flacca } \\
\text { (Dillwyn) Thuret }\end{array}$ & A & & & & & 4 & $<0.01$ \\
\hline $\begin{array}{l}\text { Ulva intestinalis } \\
\text { Linnaeus }\end{array}$ & A & 9 & $0.23 \pm 1.14$ & 44 & $0.48 \pm 2.15$ & 26 & $0.35 \pm 1.58$ \\
\hline $\begin{array}{l}\text { Urospora } \\
\text { penicilliformis (Roth) } \\
\text { Areschoug }\end{array}$ & A & & & 3 & $<0.01$ & & \\
\hline $\begin{array}{l}\text { Amphibalanus } \\
\text { improvisus Darwin }\end{array}$ & $\mathrm{S}$ & 30 & $0.55 \pm 1.47$ & 18 & $0.65 \pm 1.96$ & 5 & $0.07 \pm 0.45$ \\
\hline $\begin{array}{l}\text { Asellus aquaticus } \\
\text { (Linnaeus) }\end{array}$ & $\mathrm{H}$ & 5 & $<0.01$ & 3 & $<0.01$ & 11 & $<0.01$ \\
\hline $\begin{array}{l}\text { Bithynia tentaculata } \\
\text { (Linnaeus) }\end{array}$ & $\mathrm{H}$ & 7 & $0.04 \pm 0.18$ & 18 & $0.32 \pm 1.06$ & 1 & $<0.01$ \\
\hline $\begin{array}{l}\text { Cerastoderma glaucum } \\
\text { (Poiret) }\end{array}$ & $\mathrm{S}$ & 25 & $0.28 \pm 1.28$ & 71 & $0.98 \pm 2.03$ & 57 & $1.20 \pm 2.59$ \\
\hline Family Chironomidae & $\mathrm{D}$ & 55 & $0.05 \pm 0.27$ & 62 & $0.16 \pm 0.53$ & 67 & $0.02 \pm 0.05$ \\
\hline Order Coleoptera & $\mathrm{C}$ & 2 & $<0.01$ & 15 & $<0.01$ & 1 & $<0.01$ \\
\hline $\begin{array}{l}\text { Cordylophora caspia } \\
\text { (Pallas) }\end{array}$ & S & & & 3 & $0.04 \pm 0.21$ & & \\
\hline Corixa Geoffroy & $\mathrm{C}$ & & & 3 & $<0.01$ & 3 & $<0.01$ \\
\hline $\begin{array}{l}\text { Corophium volutator } \\
\text { (Pallas) }\end{array}$ & $\mathrm{D}$ & 2 & $0.02 \pm 0.11$ & 18 & $0.06 \pm 0.24$ & 25 & $0.01 \pm 0.08$ \\
\hline $\begin{array}{l}\text { Cyanophthalma } \\
\text { obscura (Schultze) }\end{array}$ & $\mathrm{C}$ & 11 & $<0.01$ & 18 & $<0.01$ & 30 & $<0.01$ \\
\hline Order Diptera & $\mathrm{C}$ & & & 3 & $<0.01$ & 1 & $<0.01$ \\
\hline $\begin{array}{l}\text { Dreissena polymorpha } \\
\text { (Pallas) }\end{array}$ & $\mathrm{S}$ & & & 47 & $6.44 \pm 16.38$ & & \\
\hline $\begin{array}{l}\text { Ecrobia ventrosa } \\
\text { (Montagu) }\end{array}$ & $\mathrm{H}$ & 14 & $0.01 \pm 0.06$ & 15 & $0.09 \pm 0.45$ & 33 & $0.44 \pm 1.57$ \\
\hline $\begin{array}{l}\text { Gammarus duebeni } \\
\text { Liljeborg }\end{array}$ & $\mathrm{H}$ & & & 9 & $<0.01$ & 1 & $<0.01$ \\
\hline $\begin{array}{l}\text { Gammarus juv. } \\
\text { Fabricius }\end{array}$ & $\mathrm{H}$ & 86 & $0.24 \pm 0.36$ & 82 & $0.14 \pm 0.22$ & 84 & $0.15 \pm 0.31$ \\
\hline $\begin{array}{l}\text { Gammarus locusta } \\
\text { (Linnaeus) }\end{array}$ & $\mathrm{H}$ & & & & & 5 & $0.02 \pm 0.14$ \\
\hline
\end{tabular}


Table 2. Continued

\begin{tabular}{|c|c|c|c|c|c|c|c|}
\hline \multirow[t]{2}{*}{ Taxon } & \multirow[t]{2}{*}{ Function } & \multicolumn{2}{|r|}{ Eru } & \multicolumn{2}{|r|}{ Liu } & \multicolumn{2}{|r|}{ Kõiguste } \\
\hline & & $\%$ & $\mathrm{~B}$ & $\%$ & $\mathrm{~B}$ & $\%$ & $\mathrm{~B}$ \\
\hline $\begin{array}{l}\text { Gammarus oceanicus } \\
\text { Segerstråle }\end{array}$ & $\mathrm{H}$ & 91 & $1.20 \pm 1.78$ & 15 & $<0.01$ & 49 & $0.07 \pm 0.17$ \\
\hline $\begin{array}{l}\text { Gammarus salinus } \\
\text { Spooner }\end{array}$ & $\mathrm{H}$ & 61 & $0.05 \pm 0.13$ & 47 & $0.06 \pm 0.12$ & 57 & $0.07 \pm 0.11$ \\
\hline Gammarus spp. & $\mathrm{H}$ & & & & & 5 & $0.05 \pm 0.36$ \\
\hline $\begin{array}{l}\text { Gammarus zaddachi } \\
\text { Sexton }\end{array}$ & $\mathrm{H}$ & 5 & $<0.01$ & 12 & $<0.01$ & 7 & $0.01 \pm 0.08$ \\
\hline $\begin{array}{l}\text { Hediste diversicolor } \\
\text { (O. F. Müller) }\end{array}$ & $\mathrm{D}$ & 5 & $<0.01$ & 35 & $0.02 \pm 0.05$ & 43 & $0.07 \pm 0.16$ \\
\hline Subclass Hirudinea & $\mathrm{C}$ & & & & & 1 & $<0.01$ \\
\hline Hydrobia spp. & $\mathrm{H}$ & 27 & $0.03 \pm 0.10$ & 32 & $0.10 \pm 0.23$ & 43 & $0.81 \pm 3.39$ \\
\hline Idotea balthica (Pallas) & $\mathrm{H}$ & 59 & $1.30 \pm 2.96$ & 9 & $0.03 \pm 0.16$ & 39 & $0.05 \pm 0.13$ \\
\hline Idotea chelipes (Pallas) & $\mathrm{H}$ & 52 & $0.20 \pm 0.99$ & 56 & $0.07 \pm 0.15$ & 49 & $0.04 \pm 0.10$ \\
\hline $\begin{array}{l}\text { Idotea granulosa } \\
\text { Rathke }\end{array}$ & $\mathrm{H}$ & 9 & $0.03 \pm 0.11$ & & & 1 & $<0.01$ \\
\hline Jaera albifrons Leach & $\mathrm{H}$ & 59 & $0.01 \pm 0.03$ & 21 & $<0.01$ & 62 & $0.02 \pm 0.03$ \\
\hline Order Lepidoptera & $\mathrm{C}$ & & & 24 & $0.01 \pm 0.04$ & 5 & $<0.01$ \\
\hline $\begin{array}{l}\text { Leptocheirus pilosus } \\
\text { Zaddach }\end{array}$ & $\mathrm{D}$ & & & 3 & $<0.01$ & 3 & $<0.01$ \\
\hline $\begin{array}{l}\text { Lymnaea peregra } \\
\text { (Müller) }\end{array}$ & $\mathrm{H}$ & 82 & $0.97 \pm 1.45$ & 24 & $0.09 \pm 0.36$ & 42 & $0.49 \pm 1.56$ \\
\hline Lymnaea spp. & $\mathrm{H}$ & 2 & $<0.01$ & 3 & $0.10 \pm 0.56$ & 3 & $0.01 \pm 0.08$ \\
\hline $\begin{array}{l}\text { Macoma balthica } \\
\text { (Linnaeus) }\end{array}$ & $\mathrm{D}$ & 9 & $<0.01$ & 53 & $2.97 \pm 8.75$ & 54 & $1.80 \pm 4.64$ \\
\hline Mya arenaria Linnaeus & $\mathrm{S}$ & 5 & $<0.01$ & 3 & $<0.01$ & 30 & $0.60 \pm 2.96$ \\
\hline Mysis mixta Lilljeborg & $\mathrm{C}$ & 2 & $<0.01$ & & & & \\
\hline Mytilus trossulus Gould & $\mathrm{S}$ & 39 & $3.85 \pm 12.95$ & 6 & $<0.01$ & 91 & $24.70 \pm 40.49$ \\
\hline $\begin{array}{l}\text { Neomysis integer } \\
\text { (Leach) }\end{array}$ & $\mathrm{C}$ & & & 12 & $<0.01$ & 4 & $<0.01$ \\
\hline Order Odonata & $\mathrm{C}$ & & & 15 & $0.01 \pm 0.04$ & 3 & $<0.01$ \\
\hline Subclass Oligochaeta & $\mathrm{D}$ & 2 & $<0.01$ & & & 8 & $<0.01$ \\
\hline $\begin{array}{l}\text { Peringia ulvae } \\
\text { (Pennant) }\end{array}$ & $\mathrm{H}$ & 11 & $0.02 \pm 0.07$ & 9 & $0.01 \pm 0.03$ & 51 & $0.66 \pm 1.43$ \\
\hline $\begin{array}{l}\text { Physa fontinalis } \\
\text { (Linnaeus) }\end{array}$ & $\mathrm{H}$ & 7 & $<0.01$ & & & 7 & $0.04 \pm 0.21$ \\
\hline $\begin{array}{l}\text { Piscicola geometra } \\
\text { (Linnaeus) }\end{array}$ & $\mathrm{C}$ & 2 & $<0.01$ & 15 & $<0.01$ & 4 & $<0.01$ \\
\hline Order Plecoptera & $\mathrm{C}$ & & & & & 1 & $<0.01$ \\
\hline $\begin{array}{l}\text { Potamopygrus } \\
\text { antipodarum (Gray) }\end{array}$ & $\mathrm{H}$ & 2 & $<0.01$ & 3 & $<0.01$ & 1 & $<0.01$ \\
\hline $\begin{array}{l}\text { Praunus flexuosus } \\
\text { (Müller) }\end{array}$ & $\mathrm{C}$ & & & & & 1 & $<0.01$ \\
\hline $\begin{array}{l}\text { Saduria entomon } \\
\text { (Linnaeus) }\end{array}$ & $\mathrm{C}$ & 5 & $0.02 \pm 0.11$ & 6 & $0.06 \pm 0.34$ & 17 & $0.02 \pm 0.13$ \\
\hline $\begin{array}{l}\text { Theodoxus fluviatilis } \\
\text { (L.) }\end{array}$ & $\mathrm{H}$ & 98 & $7.87 \pm 10.08$ & 76 & $1.43 \pm 3.53$ & 82 & $2.95 \pm 5.03$ \\
\hline Order Trichoptera & $\mathrm{C}$ & 2 & $0.01 \pm 0.08$ & 3 & $<0.01$ & 7 & $<0.01$ \\
\hline
\end{tabular}


Relationship between nutrient loading and benthos

functional groups but not the biomass of P. littoralis and carnivores (Table 3). The BRT modelling showed that nutrient load strongly correlated to species richness and biomass of macroalgal and invertebrate species and functions. In general elevated nutrient loads increased the species richness of macrophytes and benthic invertebrates, the biomass of annual macroalgae and of the majority of invertebrate feeding groups, and reduced the biomass of perennial macroalgae. The biomasses of the majority of species and functions were a function of the gulf-scale nutrient loading whereas the dynamics of annual algae and chironomidae reflected changes in water-body level nutrient loading (Table 3, Figs 2-5).

With elevating nutrient loads $F$. vesiculosus initially increased its biomass but then at a certain threshold level its biomass drastically declined. The invertebrate species associated with $F$. vesiculosus (such as Gammarus oceanicus, Idotea balthica, and T. fluviatilis) showed exactly the same response pattern along the nutrient gradient. Among the studied species the variability in the biomass of

Table 3. The percentage of variance explained (\% var) and relative contribution of different environmental variables (D - sampling depth, B - bottom substrate, $\mathrm{S}$ - water salinity, GULF-N - annual gulf-scale nitrogen loading, GULF-P - annual gulf-scale phosphorus loading, WB-N - annual water-body level nitrogen loading, WB-P - annual water-body level phosphorus loading) to the BRT models based on the biomasses and richness of macrophyte and invertebrate species and functional groups

\begin{tabular}{l|c|r|c|r|r|r|r|r}
\hline \multicolumn{1}{c}{$\begin{array}{c}\text { Species or } \\
\text { functional group }\end{array}$} & \% var & D & B & S & GULF-N & GULF-P & WB-N & WB-P \\
\hline Ceramium tenuicorne & 69.1 & 33.9 & 0.0 & 15.0 & 12.2 & 18.4 & 10.5 & 10.0 \\
Cladophora glomerata & 96.7 & 26.6 & 0.0 & 15.0 & 15.7 & 6.3 & 27.5 & 8.9 \\
Pilayella littoralis & 45.8 & 38.8 & 0.0 & 4.4 & 31.3 & 8.8 & 3.9 & 12.8 \\
Furcellaria lumbricalis & 71.4 & 39.8 & 0.0 & 6.9 & 2.9 & 9.4 & 26.5 & 14.5 \\
Fucus vesiculosus & 57.7 & 9.1 & 0.0 & 1.1 & 85.9 & 0.2 & 1.6 & 2.1 \\
Myriophyllum spicatum & 77.4 & 9.6 & 0.3 & 37.3 & 4.0 & 1.1 & 33.6 & 14.1 \\
Cerastoderma glaucum & 66.9 & 32.6 & 0.5 & 21.1 & 7.1 & 6.4 & 9.2 & 23.1 \\
Chironomidae & 68.3 & 25.7 & 2.1 & 7.7 & 7.1 & 11.4 & 25.3 & 20.7 \\
Gammarus salinus & 70.5 & 35.4 & 0.0 & 9.9 & 22.5 & 12.6 & 11.5 & 8.1 \\
Gammarus oceanicus & 67.8 & 14.4 & 0.0 & 3.6 & 66.7 & 7.1 & 4.8 & 3.4 \\
Idotea balthica & 83.9 & 17.5 & 0.0 & 2.9 & 45.4 & 21.3 & 6.9 & 6.0 \\
Theodoxus fluviatilis & 93.7 & 29.1 & 0.0 & 8.2 & 34.2 & 10.8 & 9.5 & 8.2 \\
Annual macrophytes & 67.8 & 6.7 & 0.0 & 43.0 & 5.0 & 0.8 & 30.0 & 14.5 \\
Perennial macrophytes & 56.4 & 10.9 & 0.0 & 5.9 & 75.3 & 1.7 & 2.9 & 3.3 \\
Total macrophytes & 63 & 44.3 & 0.0 & 1.3 & 40.1 & 1.1 & 9.0 & 4.2 \\
Deposit feeders & 80.6 & 16.5 & 0.0 & 9.0 & 34.7 & 2.7 & 27.7 & 9.4 \\
Suspension feeders & 66.2 & 51.1 & 0.0 & 3.3 & 28.4 & 14.2 & 1.2 & 1.8 \\
Herbivores & 80.7 & 31.2 & 0.0 & 9.9 & 20.7 & 16.4 & 11.7 & 10.1 \\
Carnivores & 32.7 & 6.7 & 0.0 & 6.0 & 15.2 & 1.9 & 47.7 & 22.5 \\
Total invertebrates & 66.6 & 40.4 & 0.0 & 4.1 & 16.9 & 17.9 & 9.8 & 10.9 \\
Macrophyte richness & 87.9 & 28.4 & 0.0 & 12.1 & 17.3 & 8.9 & 19.4 & 13.9 \\
Invertebrate richness & 75.9 & 33.9 & 0.0 & 8.9 & 12.5 & 18.5 & 16.2 & 10.0
\end{tabular}



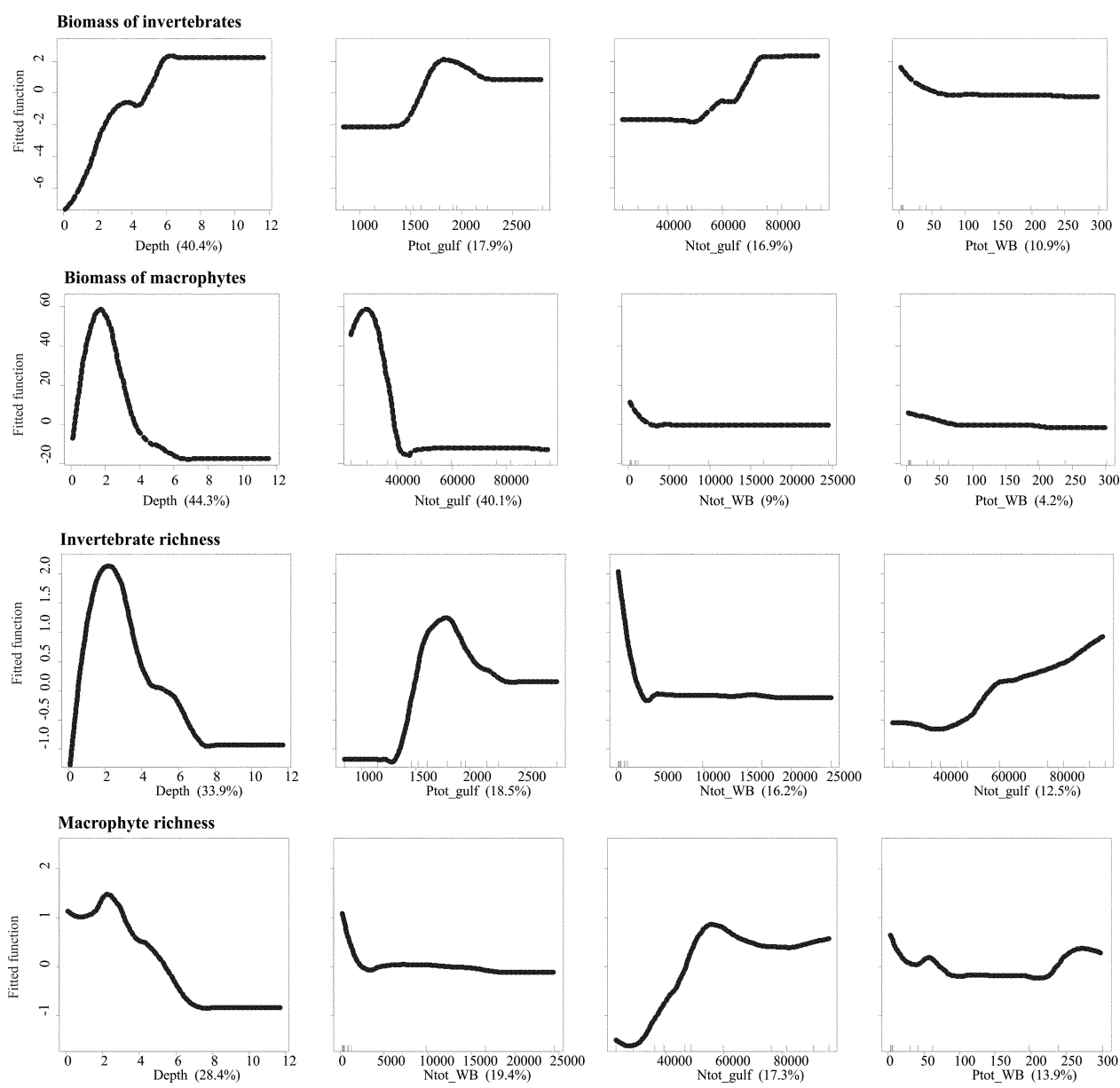

Fig. 2. Partial dependence plots showing the effect of environmental variables on the total biomass and species richness of benthic invertebrates and macrophytes whilst all other variables are held at their means. Ntot and Ptot denote annual total nitrogen and phosphorus loads; WB denotes waterbody and gulf basin scale. Depth is the sampling depth and salinity is the annual average of the studied transect area.

M. spicatum was to the largest extent affected by water salinity but also water-body level nutrient loading was an important variable for this species (Figs 2-5).

In addition to many strong direct effects, often environmental variables interactively contributed to the biotic patterns. Nevertheless, other than twoway interactions had weak effects on the studied response variables. In general nutrient loading affected interactively with salinity and depth the biomass and diversity of benthic macrophytes and invertebrates. Surprisingly, sediment type had no interactive effects with any studied environmental variable on the biotic patterns (Table 4). 

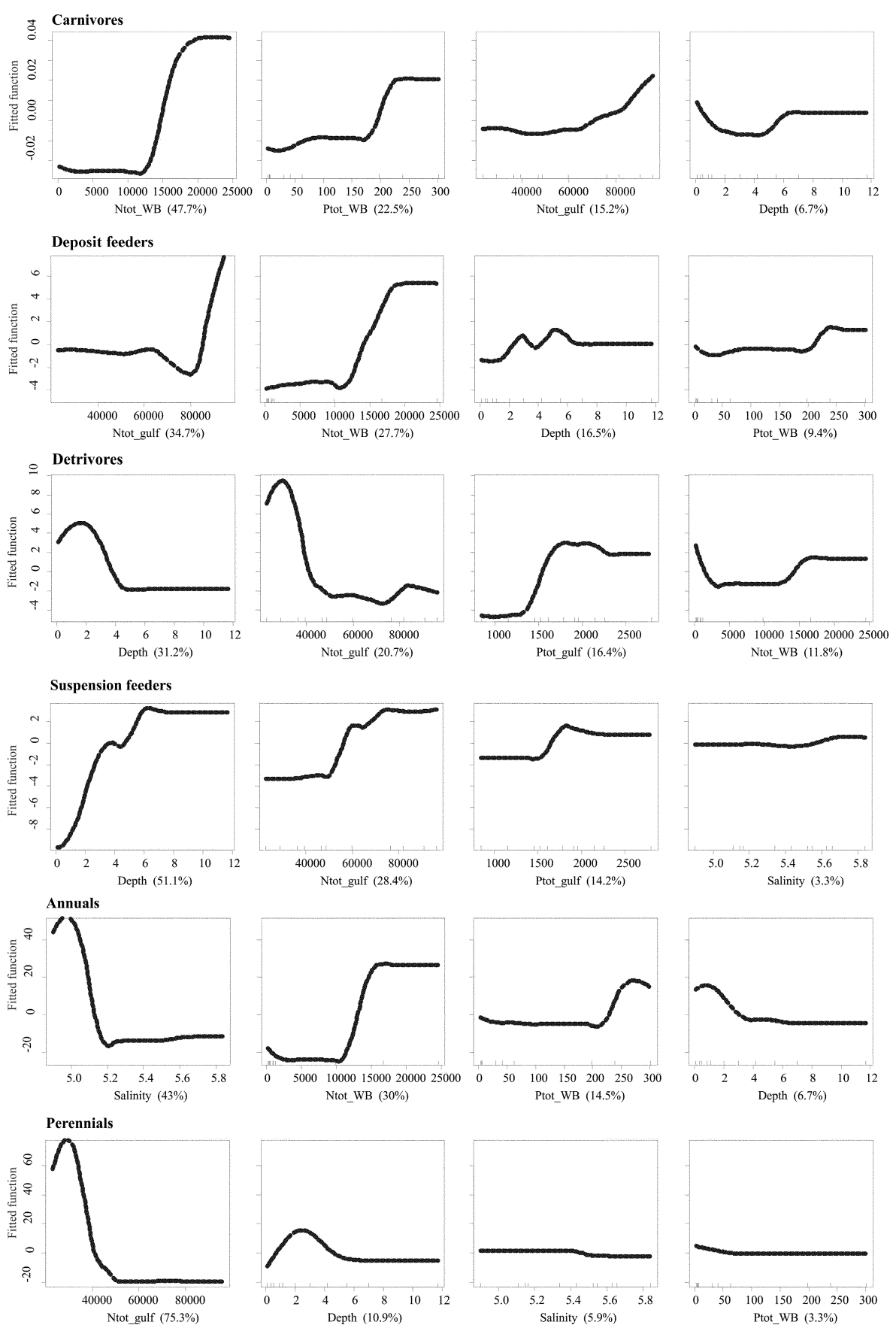

Fig. 3. Partial dependence plots showing the effect of environmental variables on the biomass of invertebrate and macrophyte functions (for invertebrates: carnivores, deposit feeders, herbivores, suspension feeders, and macroalgae: annuals, perennials) whilst all other variables are held at their means. Ntot and Ptot denote annual total nitrogen and phosphorus loads; WB denotes water-body and gulf basin scale. Depth is the sampling depth and salinity is the annual average of the studied transect area. 

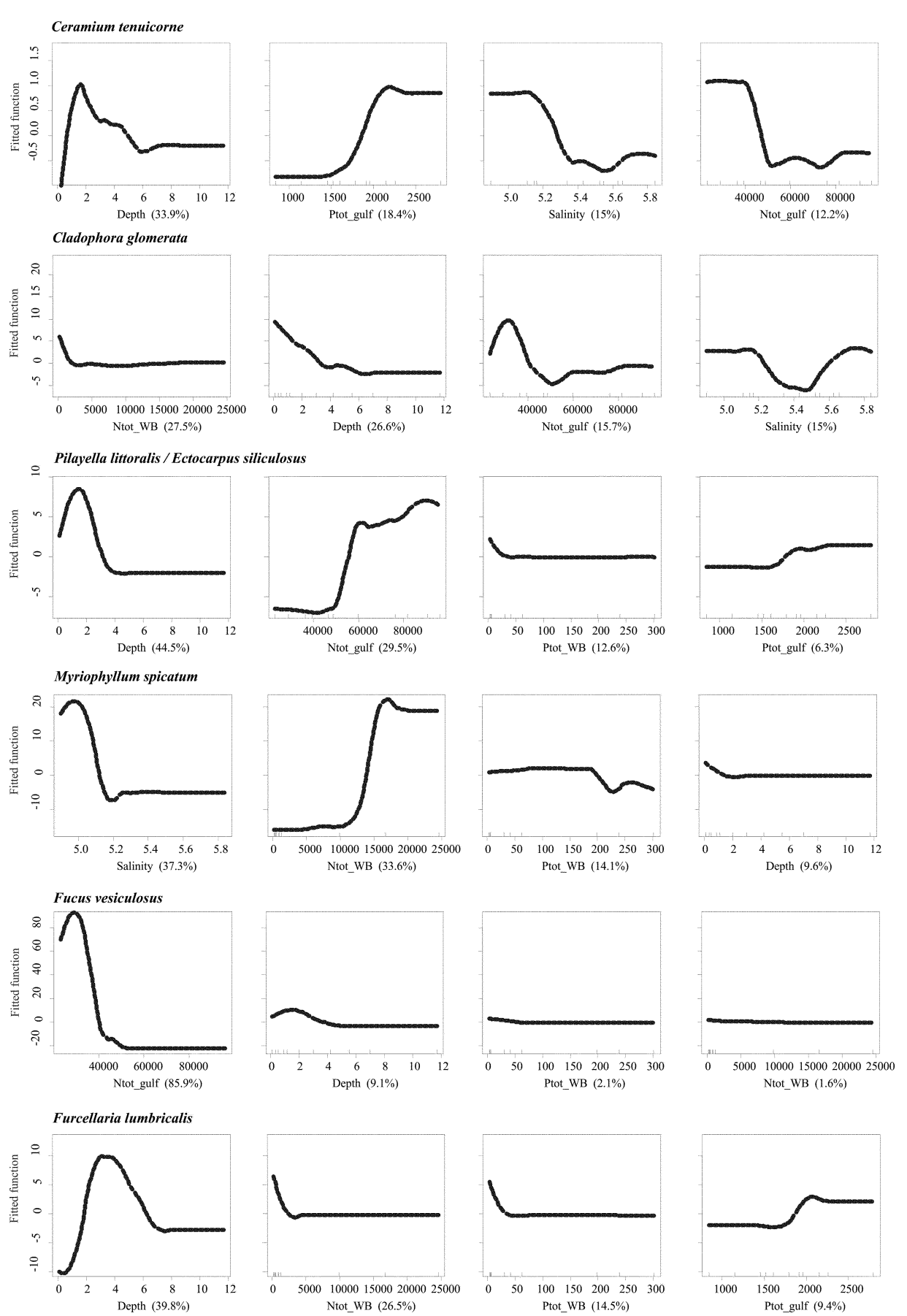

Fig. 4. Partial dependence plots showing the effect of environmental variables on the biomass of Ceramium tenuicorne, Cladophora glomerata, Pilayella littoralis/Ectocarpus siliculosus, Myriophyllum spicatum, Fucus vesiculosus, and Furcellaria lumbricalis. Ntot and Ptot denote annual total nitrogen and phosphorus loads; WB denotes water-body and gulf basin scale. Depth is the sampling depth and salinity is the annual average of the studied transect area. 

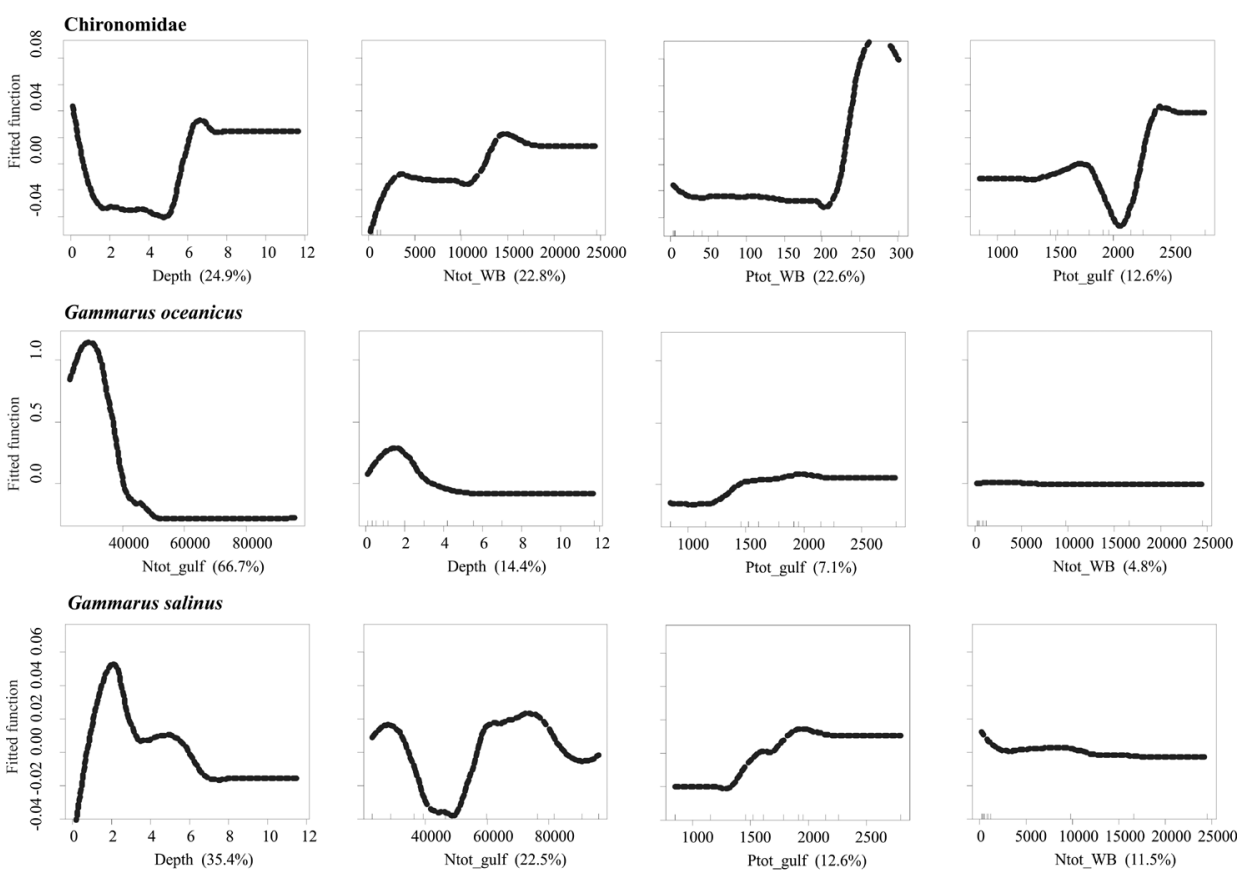

Idotea balthica
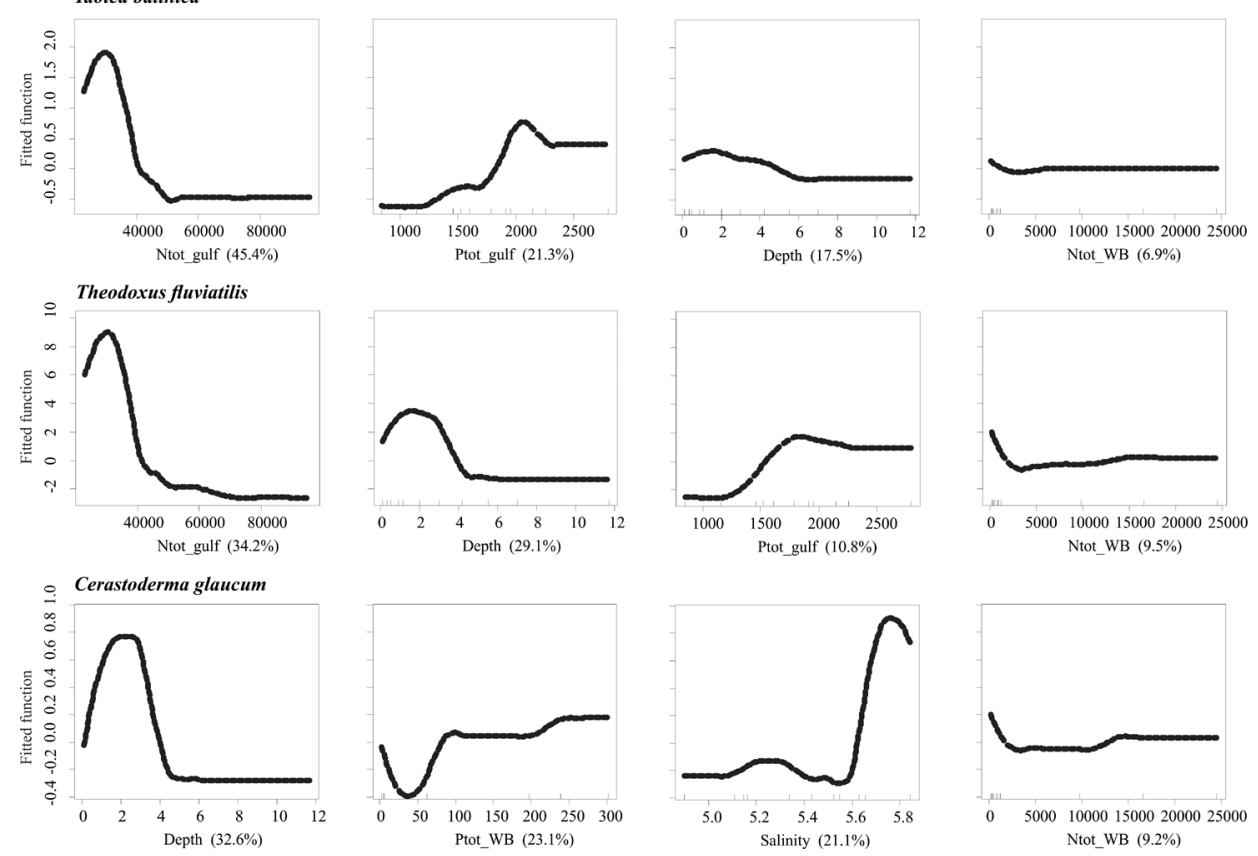

Fig. 5. Partial dependence plots showing the effect of environmental variables on the biomass of Chironomidae, Gammarus oceanicus, Gammarus salinus, Idotea balthica, Theodoxus fluviatilis, and Cerastoderma glaucum. Ntot and Ptot denote annual total nitrogen and phosphorus loads; WB denotes water-body and gulf basin scale. Depth is the sampling depth and salinity is the annual average of the studied transect area. 
Table 4. Relative contribution of two-way interactions (\%) of the studied environmental variables on the biomass and richness of key macrophyte and invertebrate functional groups. The codes of environmental variables are as follows: Depth - sampling depth, Bottom - bottom substrate, GULF-N - annual gulf-scale nitrogen loading, GULF-P - annual gulf-scale phosphorus loading, WB-N - annual water-body level nitrogen loading, WB-P - annual water-body level phosphorus loading, Salinity - water salinity

\begin{tabular}{l|r|r|r|r|r|r}
\hline & Bottom & Gulf-N & Gulf-P & WB-N & WB-P & Salinity \\
\hline$\quad$ Total macrophyte biomass & & & & & & \\
Depth & 0 & 31 & 4 & 4 & 28 & 3 \\
Bottom & & 0 & 0 & 0 & 0 & 0 \\
Gulf-N & & & 20 & 2 & 3 & 3 \\
Gulf-P & & & & 0 & 2 & 0 \\
WB-N & & & & & 0 & 0 \\
WB-P & & & & & & 0
\end{tabular}

Total invertebrate biomass

Depth

Bottom

Gulf-N

Gulf-P

WB-N

WB-P

Annual algal biomass

Depth

Bottom

Gulf-N

Gulf-P

WB-N

WB-P

Perennial algal biomass

Depth

Bottom

Gulf-N

Gulf-P

WB-N

WB-P

Deposit feeder biomass

Depth
Bottom
Gulf-N
Gulf-P
WB-N
WB-P

Suspension feeder biomass

Depth

Bottom

Gulf-N

Gulf-P

WB-N

WB-P

$\begin{array}{rrrrrr}0 & 71 & 15 & 1 & 1 & 1 \\ & 0 & 0 & 0 & 0 & 0 \\ & 2 & 0 & 0 & 4 \\ & & 2 & 4 & 0 \\ & & & 0 & 0 \\ & & & & \end{array}$

$\begin{array}{rrrrrr}0 & 20 & 0 & 19 & 18 & 6 \\ & 0 & 0 & 0 & 0 & 0 \\ & & 0 & 10 & 0 & 0 \\ & & 3 & 1 & 7 \\ & & & 4 & 3 \\ & & & & 8\end{array}$

$\begin{array}{rrrrrr}0 & 31 & 1 & 5 & 2 & 1 \\ & 0 & 0 & 0 & 0 & 0 \\ & 22 & 14 & 4 & 19 \\ & & 1 & 0 & 0 \\ & & & 0 & 0 \\ & & & & \end{array}$

$\begin{array}{rrrrrr}0 & 28 & 4 & 34 & 3 & 4 \\ & 0 & 0 & 0 & 0 & 0 \\ & 1 & 14 & 3 & 7 \\ & & 1 & 0 & 0 \\ & & & 0 & 1 \\ & & & & \end{array}$

$\begin{array}{rrrrrr}0 & 79 & 15 & 0 & 1 & 1 \\ & 0 & 0 & 0 & 0 & 0 \\ & 1 & 0 & 2 & 0 \\ & & 0 & 0 & 1 \\ & & & 0 & 0 \\ & & & & \end{array}$


Relationship between nutrient loading and benthos

Table 4. Continued

\begin{tabular}{|c|c|c|c|c|c|c|}
\hline & Bottom & Gulf-N & Gulf-P & WB-N & WB-P & Salinity \\
\hline \multicolumn{7}{|c|}{ Herbivore biomass } \\
\hline Depth & 0 & 35 & 26 & 2 & 4 & 2 \\
\hline Bottom & & 0 & 0 & 0 & 0 & 0 \\
\hline Gulf-N & & & 25 & 1 & 1 & 3 \\
\hline Gulf-P & & & & 0 & 0 & 0 \\
\hline WB-N & & & & & 0 & 1 \\
\hline WB-P & & & & & & 0 \\
\hline \multicolumn{7}{|c|}{ Macrophyte taxonomic richness } \\
\hline Depth & 0 & 54 & 4 & 12 & 9 & 5 \\
\hline Bottom & & 0 & 0 & 0 & 0 & 0 \\
\hline Gulf-N & & & 11 & 0 & 0 & 1 \\
\hline Gulf-P & & & & 0 & 1 & 0 \\
\hline WB-N & & & & & 1 & 1 \\
\hline WB-P & & & & & & 2 \\
\hline \multicolumn{7}{|c|}{ Invertebrate taxonomic richness } \\
\hline Depth & 0 & 46 & 3 & 13 & 10 & 2 \\
\hline Bottom & & 0 & 0 & 0 & 0 & 0 \\
\hline Gulf-N & & & 9 & 2 & 1 & 1 \\
\hline Gulf-P & & & & 5 & 5 & 2 \\
\hline WB-N & & & & & 0 & 0 \\
\hline WB-P & & & & & & 1 \\
\hline
\end{tabular}

\section{DISCUSSION}

The study showed that nutrient load strongly correlated with the diversity and biomass of macroalgal and invertebrate species but mainly was in interaction with local abiotic variables such as salinity and depth. Salinity is regarded as the key environmental variable determining the large-scale distribution of macrophytes and benthic invertebrates in the Baltic Sea basin (Kautsky and van der Maarel, 1990; Bonsdorff and Pearson, 1999), and fluctuation in salinity close to the salinity tolerance limit may override the effect of nutrient loads (Josefson and Hansen, 2004; Krause-Jensen et al., 2007a, 2007b). At the local scale salinity conditions have a strong structuring role especially in estuaries where the salinity conditions fluctuate within a greater range. Owing to the irregular freshwater loading by the Pärnu River salinity is expected to have stronger effects in the Liu area compared to the other studied sites. The availability of light is known to play a crucial role in regulating primary production of macroalgae (Field et al., 1998); therefore variation in depth (i.e. a proxy of the light regime) is expected to largely contribute to the patterns of macroalgal species. Among the studied macrophyte and invertebrate species $M$. spicatum was the most sensitive to changes in salinity with a clear reduction in its biomass above 5.2 PSU. This species is known to have a depressed photosynthesis at elevated salinities resulting in a reduced ratio of photosynthesis to respiration and lower biomass standing stock (McGahee and Davis, 1971). 
In general, higher nutrient loads increased the species richness and biomass of annual macroalgae and reduced the biomass of perennial macroalgae. As such the results confirm our expectations and corroborate earlier findings (e.g. Field et al., 1998). Nutrient loading is considered as a key factor structuring the phytobenthos communities in the Baltic Sea area (e.g. Haahtela, 1984; Hällfors et al., 1984; Havens et al., 2001). An increase of nutrient concentrations is often accompanied by an increase of opportunistic filamentous algae (e.g. Wallentinus, 1984a; Kautsky et al., 1988; Mäkinen et al., 1988; Pedersen, 1995; Morand and Briand, 1996). Similarly, experimental studies have demonstrated that nutrients stimulate the growth of ephemeral algae (Pedersen and Borum, 1996; Bokn et al., 2003). Such relationship is explained by the higher nutrient uptake of annual algae compared to perennial algae at high nutrient concentrations (Wallentinus, 1984a, 1984b).

In our study the magnitude of the effect of nutrients on macrophytes was highly species-specific. Similarly, the functional form relationship between nutrients and macrophytes varied among macroalgal species and cannot be easily generalized.

In the main the variability in macrophyte biomasses was affected by the gulfscale nutrient loading but in some cases the water-body level nutrient loading also contributed to the biomass variability. For example, among the ephemeral algae, $P$. littoralis seemed to benefit the most of gulf-scale nutrient enrichment. On the other hand, C. tenuicorne benefitted only from the gulf-scale phosphorus loading whereas the species was actually negatively affected by the gulf-scale nitrogen loading. Although salinity conditions do not vary largely in our study area, it has been shown that the growth rate of $C$. tenuicorne is a function of salinity and nutrients, and in different regions the species responds differently to nutrient loads (Bergström and Kautsky, 2006). This may explain the opposing effects of nitrogen and phosphorus to this red algal species. Alternatively, this opportunistic filamentous algal species may benefit from phosphorus excess.

Cladophora glomerata in turn showed a dramatic increase in its biomass at the initial increment of the gulf-scale level nutrient loading but a further increase in the gulf-scale nutrients reduced its biomass. Locally, however, an increment in the water-body level phosphorus increased the biomass of $C$. glomerata while that of nitrogen reduced its biomass. Such contradicting patterns may arise from interactive effects of e.g. nutrient loading and wave exposure. Results from the mesocosm experiment by Kraufvelin et al. (2010) indicate that the biomass of red and brown filamentous algae is often smaller at higher nutrient concentrations irrespective of wave exposure, whereas the growth of green filamentous algae is favoured by higher nutrient levels and higher wave activity.

In some cases the observed negative effect of elevated nutrient loading on annual macroalgal species may be indirect, especially for those annual species that inhabit deeper areas. Namely, an increasing nutrient load is often associated with the reduced water transparency. Therefore, the reduced coverage of annual species in the deeper areas may be attributed to the poor light climate rather than the direct effect of nutrient loading (Krause-Jensen et al., 2007b). Besides the direct effect of light conditions, the algae growing at suboptimal light become more attractive to herbivores (Geertz-Hansen et al., 1993; Paalme et al., 2002; 
Kotta et al., 2006). In our study only the linkage between annual nutrient loads and biotic patterns was examined and we did not discern the effect of nutrient limitation during the growth season. Such cause-effect patterns can be disentangled only if an experimental framework is used.

Although an increase in annual macroalgal species is a general pattern observed worldwide, it has been shown in some observational and experimental studies that there is no clear link between the nutrient loading and biomasses of annual and perennial species (Pihl et al., 1999; Karez et al., 2004). Nevertheless, our study clearly showed that both annual and perennial algae captured the shifts in regional nutrient loads and therefore might be rewarding elements for the water quality assessment in the north-eastern Baltic Sea.

The effect of nutrients did not vary largely among different invertebrate functional groups. In general moderate nutrient loads increased the biomass of invertebrate feeding groups. This suggests that the studied invertebrates are not very selective in their dietary requirements. Together with the increment of nutrient loading invertebrates benefit from the improved availability of food such as organic debris, micro- and/or macroalgae.

At the species level an increment of nutrient loading increased the biomasses of the majority of benthic invertebrates. With further increment in nutrient loads species associated with the $F$. vesiculosus communities showed exactly the same response pattern along the nutrient gradient, i.e. a decline in their biomass. It is likely that this is not a direct effect of nutrients on invertebrate species but rather reflects their responses to the structural and functional changes in macrophyte communities. Vegetation is crucial for herbivores as well as for other trophic guilds either as a food source or as protection against predators (e.g. Puttman, 1986; Gee and Warwick, 1994; Pavia et al., 1999; Kotta et al., 2000). Such facilitative effect is also demonstrated by strong positive correlations between the biomass of algal species and the biomass of herbivores (Kotta and Orav, 2001; Lauringson and Kotta, 2006). Although herbivores respond to changes in total biomass of macroalgae they are highly selective among macroalgal species (Kotta et al., 2004; Orav-Kotta and Kotta, 2004; Orav-Kotta et al., 2009).

Substrate is also considered as an important structuring factor for the phytobenthic and benthic invertebrate communities (Kautsky, 1988; Kautsky et al., 1999; Kotta et al., 2007). In our models substrate was not an important explaining variable. Our study area is characterized by a mosaic of substrate at small spatial scales, i.e. practically all substrate types are available within a $100 \mathrm{~m}$ range. Thus, substrate is most likely not limiting the distribution of macrophyte and benthic invertebrate species in our transect area.

The BRT models performed poorly when modelling the biomass of $P$. littoralis and carnivores. This is probably due to the strong signal of seasonality not taken into account in the current study and/or the low representation of carnivores in the samples.

To conclude, the species richness and biomasses of macrophyte and benthic invertebrate species and functions were explained by multiple environmental factors with nutrient loading being one of the key predictors in the models. 
Although the magnitude, direction, and functional form relationship of effects varied among species and functional groups, higher nutrient loadings clearly benefitted ephemeral algae and the majority of benthic invertebrate feeding groups but had a negative effect on perennial macrophytes. The study also showed that the effect of nutrient loading was scale-specific, i.e. different responses to nutrients were observed at water-body and gulf scales. The study confirmed the biomasses of annual and perennial macrophyte species to be a rewarding element for the water quality assessment in the north-eastern Baltic Sea in case depth and salinity information is taken into account.

\section{ACKNOWLEDGEMENTS}

Funding for this research was provided by Institutional research funding IUT02-20 of the Estonian Research Council. The study was partly supported by the projects 'EstKliima' No. 3.2.0802.11-0043 and 'The status of marine biodiversity and its potential futures in the Estonian coastal sea' No. 3.2.0801.11-0029 of the Environmental Protection and Technology Programme of the European Regional Fund.

\section{REFERENCES}

Bendtsen, J., Gustafsson, K. E., Söderkvist, J., and Hansen, J. L. S. 2009. Ventilation of bottom water in the North Sea-Baltic Sea transition zone. Journal of Marine Systems, 75, 138-149.

Bergström, L. and Kautsky, L. 2006. Local adaptation of Ceramium tenuicorne (Ceramiales, Rhodophyta) within the Baltic Sea. Journal of Phycology, 42, 36-42.

Bokn, T. L., Duarte, C. M., Pedersen, M. F., Marbà, N., Moy, F. E., Barrón, C., and Bjerkeng, B. 2003. The response of experimental rocky shore communities to nutrient additions. Ecosystems, 6, 577-594.

Bonsdorff, E. and Pearson, T. H. 1999. Variation in the sublittoral macrozoobenthos of the Baltic Sea along environmental gradients: a functional-group approach. Australian Journal of Ecology, 24, 312-326.

Bonsdorff, E., Rönnberg, C., and Aarnio, K. 2002. Some ecological properties in relation to eutrophication in the Baltic Sea. Hydrobiologia, 475/476, 371-377.

Clarke, K. R. 1993. Non-parametric multivariate analyses of changes in community structure. Australian Journal of Ecology, 18, 117-143.

Clarke, K. R. and Gorley, R. N. 2006. Primer v6. User Manual/Tutorial. Primer-E, Plymouth.

Clarke, K. R. and Green, R. H. 1988. Statistical design and analysis for a biological effects study. Marine Ecology Progress Series, 46, 213-226.

Clarke, K. R., Somerfield, P. J., and Chapman, M. G. 2006. On resemblance measures for ecological studies, including taxonomic dissimilarities and a zero-adjusted Bray-Curtis coefficient for denuded assemblages. Journal of Experimental Marine Biology and Ecology, 330, 55-80.

Duarte, C. D. 1995. Submerged aquatic vegetation in relation to different nutrient regimes. Ophelia, 41, 87-112.

Elith, J., Leathwick, J. R., and Hastie, T. 2008. A working guide to boosted regression trees. Journal of Animal Ecology, 77, 802-813.

Elmgren, R. 1989. Man's impact on the ecosystem of the Baltic Sea: energy flows today and at the turn of the century. Ambio, 18, 326-332. 
Elmgren, R. 2001. Understanding human impact on the Baltic ecosystem: changing views in recent decades. Ambio, 30, 222-231.

Eriksson, B. K. and Bergström, L. 2005. Local distribution patterns of macroalgae in relation to environmental variables in the northern Baltic Proper. Estuarine, Coastal and Shelf Science, 62, 109-117.

European Union. 2000. Directive of the European Parliament and of the Council Establishing a Framework for Community Action in the Field of Water Policy. Legislative Acts and other instruments. ENV221 CODEC 513.

Field, C. B., Behrenfeld, M. J., Randerson, J. T., and Falkowski, P. 1998. Primary production of the biosphere: integrating terrestrial and oceanic components. Science, 281, 237-240.

Gee, J. M. and Warwick, R. M. 1994. Metazoan community structure on relation to the fractal dimensions of marine algae. Marine Ecology Progress Series, 103, 141-150.

Geertz-Hansen, O., Sand-Jensen, K., Hansen, D. F., and Christiansen, A. 1993. Growth and grazing control of abundance of the marine macroalga, Ulva lactuca L. in a eutrophic Danish estuary. Aquatic Botany, 46, 101-109.

Grall, J. and Chauvaud, L. 2002. Marine eutrophication and benthos: the need for new approaches and concepts. Global Change Biology, 8, 813-830.

Granéli, E. and Sundbäck, K. 1985. The response of planktonic and microbenthic algal assemblages to nutrient enrichment in shallow coastal waters, southwest Sweden. Journal of Experimental Marine Biology and Ecology, 85, 253-268.

Gray, J. S., Wu, R. S., and Or, Y. Y. 2002. Effects of hypoxia and organic enrichment on the coastal marine environment. Marine Ecology Progress Series, 238, 249-279.

Haahtela, I. 1984. A hypothesis of the decline of the Bladder Wrack (Fucus vesiculosus L.) in SW Finland in 1975-1981. Limnologica, 15, 345-350.

Hällfors, G., Kangas, P., and Niemi, A. 1984. Recent changes in the phytal at the south coast of Finland. Ophelia, 3, 51-59.

Havens, K. E., Hauxwell, J., Tyler, A. C., Thomas, S., McGlathery, K. J., Cebrian, J., et al. 2001. Complex interactions between autotrophs in shallow marine and freshwater ecosystems: implications for community responses to nutrient stress. Environmental Pollution, 113, 95-107.

HELCOM. 2009. Manual for Marine Monitoring in the COMBINE Programme of HELCOM. Helsinki. http:/helcom.fi/action-areas/monitoring-and-assessment/manuals-and-guidelines/ combine-manual (accessed 30.07.2014).

Howarth, R. W. 1988. Nutrient limitation of net primary production in marine ecosystems. Annual Review of Ecology, Evolution, and Systematics, 19, 89-110.

Josefson, A. B. and Hansen, J. L. S. 2004. Species richness of benthic macrofauna in Danish estuaries and coastal areas. Global Ecology and Biogeography, 13, 273-288.

Karez, R., Engelbert, S., Kraufvelin, P., Pedersen, M. F., and Sommer, U. 2004. Biomass response and changes in composition of ephemeral macroalgal assemblages along an experimental gradient of nutrient enrichment. Aquatic Botany, 78, 103-117.

Kautsky, H. 1988. Factors structuring phytobenthic communities in the Baltic Sea. Doctoral thesis. Department of Zoology, University of Stockholm.

Kautsky, H. and van der Maarel, E. 1990. Multivariate approaches to the variation in phytobenthic communities and environmental vectors in the Baltic Sea. Marine Ecology Progress Series, 60, 169-184.

Kautsky, H., Kautsky, U., and Nellbring, S. 1988. Distribution of flora and fauna in an area receiving pulp mill effluents in the Baltic Sea. Ophelia, 28, 139-155.

Kautsky, H., Martin, G., Mäkinen, A., Borgiel, M., Vahteri, P., and Rissanen, J. 1999. Structure of phytobenthic and associated animal communities in the Gulf of Riga. Hydrobiologia, 393, 191-200.

Kotta, J. and Ólafsson, E. 2003. Competition for food between the introduced exotic polychaete Marenzelleria viridis and the resident native amphipod Monoporeia affinis in the Baltic Sea. Journal of Sea Research, 342, 27-35. 
Kotta, J. and Orav, H. 2001. Role of benthic macroalgae in regulating macrozoobenthic assemblages in the Väinameri (north-eastern Baltic Sea). Annales Zoologici Fennici, 38, 163-171.

Kotta, J. and Witman, J. 2009. Regional-scale patterns. In Marine Hard Bottom Communities (Wahl, M., ed.), pp. 89-99. Ecological Studies, 206. Springer-Verlag.

Kotta, J., Paalme, T., Martin, G., and Mäkinen, A. 2000. Major changes in macroalgae community composition affect the food and habitat preference of Idotea baltica. International Review of Hydrobiology, 85, 697-705.

Kotta, J., Torn, K., Martin, G., Orav-Kotta, H., and Paalme, T. 2004. Seasonal variation of invertebrate grazing on Chara connivens and C. tomentosa in Kõiguste Bay, NE Baltic Sea. Helgoland Marine Research, 58, 71-76.

Kotta, J., Orav-Kotta, H., Paalme, T., Kotta, I., and Kukk, H. 2006. Seasonal changes in situ grazing of the mesoherbivores Idotea baltica and Gammarus oceanicus on the brown algae Fucus vesiculosus and Pylaiella littoralis in the central Gulf of Finland, Baltic Sea. Hydrobiologia, 554, 117-125.

Kotta, J., Lauringson, V., and Kotta, I. 2007. Response of zoobenthic communities to changing eutrophication in the northern Baltic Sea. Hydrobiologia, 580, 97-108.

Kotta, J., Lauringson, V., Martin, G., Simm, M., Kotta, I., Herkül, K., and Ojaveer, H. 2008. Gulf of Riga and Pärnu Bay. In Ecology of Baltic Coastal Waters (Schiewer, U., ed.), pp. 217-243. Ecological Studies, 197. Springer-Verlag.

Kotta, J., Aps, R., and Orav-Kotta, H. 2009. Bayesian inference for predicting ecological water quality under different climate change scenarios. WIT Transactions on Ecology and the Environment. WIT Press, 127, 173-184.

Kraufvelin, P., Lindholm, A., Pedersen, M. F., Kirkerud, L. A., and Bonsdorff, E. 2010. Biomass, diversity and production of rocky shore macroalgae at two nutrient enrichment and wave action levels. Marine Biology, 157, 29-47.

Krause-Jensen, D., Carstensen, J., and Dahl, K. 2007a. Total and opportunistic algal cover in relation to environmental variables. Marine Pollution Bulletin, 55, 114-125.

Krause-Jensen, D., Middelboe, A., Carstensen, J., and Dahl, K. 2007b. Spatial patterns of macroalgal abundance in relation to eutrophication. Marine Biology, 152, 25-36.

Lauringson, V. and Kotta, J. 2006. Influence of the thin drift algal mats on the distribution of macrozoobenthos in Kõiguste Bay, NE Baltic Sea. Hydrobiologia, 554, 97-105.

Lauringson, V., Mälton, E., Kotta, J., Kangur, K., Orav-Kotta, H., and Kotta, I. 2007. Environmental factors influencing the biodeposition of the suspension feeding bivalve Dreissena polymorpha (Pallas): comparison of brackish and fresh water populations in the Northern Baltic Sea and Lake Peipsi. Estuarine, Coastal and Shelf Science, 75, 459-467.

Mäkinen, A., Kääriä, J., and Rajasilta, M. 1988. Factors controlling the occurrence of Furcellaria lumbricalis (Huds.) Lamour. and Phyllophora truncata (Pallas) Zinova in the upper littoral of the Archipelago of the SW Finland. Kieler Meeresforschung, 6, 140-146.

McGahee, C. F. and Davis, G. J. 1971. Photosynthesis and respiration in Myriophyllum spicatum L. as related to salinity. Limnology and Oceanography, 16, 826-829.

Morand, P. and Briand, X. 1996. Excessive growth of macroalgae: a symptom of environmental disturbance. Botanica Marina, 39, 491-516.

Orav-Kotta, H. and Kotta, J. 2004. Food and habitat choice of the isopod Idotea baltica in the northeastern Baltic Sea. Hydrobiologia, 514, 79-85.

Orav-Kotta, H., Kotta, J., Herkül, K., Kotta, I., and Paalme, T. 2009. Seasonal variability in the grazing potential of the invasive amphipod Gammarus tigrinus and the native amphipod Gammarus salinus in the northern Baltic Sea. Biological Invasions, 11, 597-608.

Paalme, T., Kukk, H., Kotta, J., and Orav, H. 2002. "In vitro" and "in situ" decomposition of nuisance macroalgae Cladophora glomerata and Pilayella littoralis. Hydrobiologia, 475/476, 469-476.

Pavia, H., Carr, H., and Aberg, P. 1999. Habitat and feeding preferences of crustacean mesoherbivores inhabiting the brown seaweed Ascophyllum nodosum (L.) Le Jol. and its epiphytic macroalgae. Journal of Experimental Marine Biology and Ecology, 236, 15-32. 
Pedersen, M. F. 1995. Nitrogen limitation of photosynthesis and growth: comparison across aquatic plant communities in a Danish estuary (Roskilde fjord). Ophelia, 41, 261-272.

Pedersen, M. F. and Borum, J. 1996. Nutrient control of algal growth in estuarine waters. Nutrient limitation and the importance of nitrogen requirements and nitrogen storage among phytoplankton and species of macroalgae. Marine Ecology Progress Series, 142, 261-272.

Pihl, L., Svenson, A., Moksnes, P.-O., and Wennhage, H. 1999. Distribution of green algal mats throughout shallow soft bottoms of the Swedish Skagerrak archipelago in relation to nutrient sources and wave exposure. Journal of Sea Research, 41, 281-294.

Posey, M. H., Alphin, T. D., Cahoon, L., Lindquist, D., and Becker, M. E. 1999. Interactive effects of nutrient additions and predation on infaunal communities. Estuaries, 22, 785-792.

Puttman, R. J. 1986. Grazing in Temperate Ecosystems: Large Herbivores and the Ecology of the New Forest. Croom Helm, London.

R Core Team. 2013. R: A Language and Environment for Statistical Computing. R Foundation for Statistical Computing, Vienna. http://www.R-project.org/ (accessed 15.12.2013).

Rönnberg, C. 2001. Effects and Consequences of Eutrophication in the Baltic Sea. Licentiate thesis, Department of Biology, Environmental and Marine Biology, Abo Akademi University, Finland.

Sand-Jensen, K. and Borum, J. 1991. Interactions among phytoplankton, periphyton and macrophytes in temperate freshwaters and estuaries. Aquatic Botany, 47, 137-175.

Valiela, I., McClelland, J., Hauxwell, J., Behr, P. J., Hersh, D., and Foreman, K. 1997. Macroalgal blooms in shallow estuaries: controls and ecophysiological and ecosystem consequences. Limnology and Oceanography, 42, 1105-1118.

Wallentinus, I. 1984a. Comparisons of nutrient uptake rates for Baltic macroalgae with different thallus morphologies. Marine Biology, 80, 215-225.

Wallentinus, I. 1984b. Partitioning of nutrient uptake between annual and perennial seaweeds in a Baltic archipelago area. Hydrobiologia, 116/117, 363-370. 